\title{
AVALIAÇÃO DA EFICÁCIA DE EXTRATOS VEGETAIS NA INIBIÇÃO DE MIRACÍDIOS DE Fasciola hepatica
}

\author{
Winner Duque Rodrigues ${ }^{1}$ \\ Larice Tosi Marques² \\ Roselena Abreu Guedes ${ }^{3}$ \\ Isabella Vilhena Freire Martins ${ }^{4}$ \\ Juliana Aparecida Severi ${ }^{5}$
}

\begin{abstract}
Resumo: A fasciolose é causada pelo trematódeo Fasciola hepatica, que se loja nos canais biliares de animais de sangue e ocasionalmente em humanos. Animais parasitados por F. hepatica apresentam retardo no desenvolvimento dentre outras enfermidades, gerando perdas econômicas consideráveis. O controle da fasciolose pode ser feito por meio do molusco hospedeiro ou por uso de fasciolicidas. No entanto, a terapêutica vigente ainda apresenta limitações de eficácia e alto custo de aquisição pelo produtor rural. Foram realizados testes in vitro com \pm 100 ovos por placa e em triplicata com o extrato vegetal a $0,5 \%$ e encubados por 14 dias em $B O D$ a $25^{\circ} \mathrm{C}$, e então, analisados sob a luz incandescente a cada hora por 3 horas. Dos sete extratos vegetais, as raízes de Harpagophytum procumbens, cascas de Uncaria tomentosa e folhas de Guapira graciliflora, Psidium guajava e de Momordica charantia apresentaram 100\% de eficácia, já as folhas de G. noxia $62,2 \%$ e de Stryphnodendron adstringens teve $95,4 \%$ contra a eclosão dos miracídios de fascíolas.
\end{abstract}

Palavras-chave: Fasciola hepatica; Produtos Naturais; Miracídios; Fasciolose; Extratos Vegetais.

\footnotetext{
1 Graduando em Farmácia pela UFES - CCENS, Alegre - ES, Brasil. E-mail: winnerduque@gmail.com

2 Mestranda em Ciências Veterinárias pela UFES - PPGCV, Brasil. E-mail: marques.larice@hotmail.com

3 Mestranda em Ciências Veterinárias pela UFES - PPGCV, Brasil. E-mail: rosinhafarma25@gmail.com

4 Professora Adjunta C-1 do Departamento de Farmácia e Nutrição - UFES - E-mail: juseveri@yahoo.com.br

5 Professora Associada do Departamento de Medicina Veterinária - UFES - E-mail: ivfmartins@gmail.com
} 